The yield of galleine by this process is quite large, and it promises, I think, to be a good improvement in the manufacture of galleine, on account of the greater cheapness of the raw materials.

N.B. After this paper had been read before the American Chemical Society, Dr. Prochazka arose and stated that he had obtained the same results as myself, about a year previous, and $I$ therefore cannot claim any originality in these experiments, but publish them merely for the benefit of the public, as Dr. Prochazka s experiments have remained hid under a bushel.

IAMES H. STEBBiNS, JR.

\title{
NOTE ON ASBESTOS FILTERS.
}

By P. Casamajor.

An extensive dealer in wares used by chemists, lately informed me that he had many inquiries concerning asbestos for filtering liquids in chemical analysis. Some chemists complain that they cannot get clear solutions through asbestos, while others, who obtain clear solutions, find that their liquids filter altogether too slowly.

Allow me to recall that the method of making asbestos filters, by pouring a thin paste of this material over a perforated platinum disc, was first proposed by me in $18: 5$, * but I necrlected to give directions concerning the preparation of asbestos, to make it fit for filtering liquids in chemical analysis. It now appears, however, that such directions would be found nseful, and I propose to repair the omission and give the necessary details.

The kind of asbestos to use is a matter of some importance. I have tried three kinds, which are sold by dealers in New York, as the Canadian, the Italian, and the Australian. This last is less flexible than the other two, and consequently the fibres do not felt together and pack as closely on the perforated plate. Hence, liquids filter more rapidly, and the Australian is, on this account, preferable to the other two kinds. I am informed that the Canadian asbestos is the most soluble in acids, but $I$ have not verified the assertion.

Whatever may be the kind of asbestos used, the following is a

\footnotetext{
* See American Chemist, V., p. 44. Chemical Neros, XXXII., p. 46.
} 
process for obtaining, with little trouble, a quantity of the pulp in a fit state for filtration:

A coarse brass sieve is placed over a sheet of paper, and a handful of asbestos is rubbed pretty roughly over the sieve cloth. This breaks it up in such a way, that the smaller fragments pass through the meshes, and are deposited on the paper underneath. After a while, the portion which remains on the sieve-cloth is collected in one bundle, and rubbed again in the same manner, and the operation is repeated until a sufficient quantity has gone through. In a few minutes, enough of the material is obtained to last for months.

As to the coarseness of mesh to use, I may say that I have used No. 10 sieve (ten openings to the inch) with satisfactory results. The sieve is best placed bottom up, so as to leave plenty of room under the cloth.

The next operation is to free the sifted material from dust and from the finest particles. This is easily accomplished by placing the asbestos, as obtained above, over another sieve of finer mesh (about No. 25 or No. 30), and stirring it while water is poured over the sieve. The first water which passes through is quite milky, but it gradually becomes clearer as the washing is continued. The washed asbestos is then put in a beaker glass, and boiled for about half an hour with strong hydrochloric acid (about 1 part of fuming HCl. to 4 parts of water).

The pulp, after this treatment, is poured over a perforated platinum plate placed in a funnel,* and washed with distilled water until no acidity is shown by litmus paper. The pulp is then taken out of the funnel and strongly beated in a platinum dish. After letting it cool sufficiently, it may be placed in a wide-mouth bottle for future use.

* A perforated platinum disc, having a stout platinum wire soldered with gold in the centre of the disc, can be used in an ordinary conical funuel. The wire finds its place in the stem of the funnel, and keeps the perforited plate in position. (See Chemical Neros, XLVI., p. 8). A Gooch crucible of sufficient size may also be used for tlee same purpose. 